\title{
26317 - A TONIC INHIBITORY CONDUCTANCE IN MURINE SPINAL NEURONS: A NOVEL SITE FOR ANESTHETICS?
}

\section{David Eng OSSD, Robert Bonin, BSc; John Macdonald, PhD; Beverley Orser, University Of Toronto, Toronto, ON, Canada}

INTRODUCTION: Anesthetic effects including immobility and analgesia are thought to be mediated via activation of GABA subtype A (GABA-A) receptors. GABA-A receptors generate two distinct forms of inhibition in the brain including conventional synaptic transmission and a tonic (persistent) inhibitory conductance via activation of extrasynaptic GABA-A receptors. ${ }^{1}$ In the hippocampus and cerebellum, extrasynaptic GABA-A receptors are highly sensitive to intravenous and inhaled anesthetics. ${ }^{1}$ It is not known whether spinal neurons also express extrasynaptic GABA-A receptors that generate a tonic conductance. The aims of this study were to determine if spinal neurons generate a tonic conductance and identify the subunit composition of the underlying GABA-A receptor(s).

METHODS: Experiments were approved by the Animal Care Committee of the University of Toronto. Primary cultures of spinal neurons were obtained from Swiss White mice and cells were incubated for 14 days. Standard patch-clamp recordings (-60 $\mathrm{mV}$ ) were performed. Neurons were continuously perfused with extracellular fluid or drug solutions using a computer-controlled multi-barreled perfusion system. Results are presented as mean \pm standard error (S.E.M). One -way ANOVA with repeated measures followed by Tukey post-hoc tests were used to determine significance $(\mathrm{p}<0.05)$ between groups.

RESULTS: The GABA-A receptor antagonist bicuculline (100 ìm) failed to alter the holding current, indicating the absence of a tonic current. The GABA-A receptor positive allosteric modulators midazolam (MIDZ, $40 \mathrm{nM}$ ) and zolpidem (ZOLP, 20 and $100 \mathrm{nM}$ ) also failed to alter the holding current. However, a low GABA concentration (1 ìm) activated a non-inactivating tonic conductance suggesting that extrasynaptic GABA-A receptors were present but that the ambient concentration of GABA in the cultures was too low to generate a tonic conductance. In the presence of 1 ìm GABA, MIDZ $(40 \mathrm{nM})$ but not ZOLP (20 and $100 \mathrm{nM}$ ) significantly enhanced the tonic current. The tonic conductance was sensitive to the classical benzodiazepine MIDZ but not the imidazopyridine benzodiazepine ZOLP, suggesting the presence of á;5 and ã subunitcontaining GABA-A receptors. Application of the GABA-A receptor agonist 4,5,6,7tetrahydroisoxazolo [5,4-c] pyridine-3-ol (THIP, $10 \mathrm{nM}$ ) activated a significant tonic current when compared to baseline levels suggesting the presence of ä; subunitcontaining GABA-A receptors.

DISCUSSION: Our results demonstrate a tonic conductance in cultured spinal neurons in the presence of low GABA concentrations. Furthermore, this conductance is likely mediated by a mixed population of extrasynaptic GABA-A receptors containing the á;5, ä;, and ã subunits. These results support the possibility that anesthetic-induced immobility and analgesia may be mediated by extrasynaptic GABA-A receptors. REFERENCES: 1. Trends Neurosci 2004 27: 262-269 